\title{
ICP monitoring in diffuse brain injury: clarifications
}

\author{
Sudish Karunakaran ${ }^{1}$
}

Received: 19 June 2018 / Accepted: 25 June 2018 / Published online: 9 July 2018

(C) Springer-Verlag GmbH Austria, part of Springer Nature 2018

Dear Editor,

I would like to congratulate the author of the submission for the critical appraisal titled 'Intracranial pressure monitoring: To do or not to do' of the article 'Intracranial pressure monitoring in diffuse brain injury: why the developing world needs it'. In response to the point-based submission, I would like to put forth the following clarifications.

The comparison is between ICP-monitored group and nonICP-monitored group in diffuse brain injury. The management protocol in the non-ICP group would remain the same in most institutions with only variations of dosage off the anti-oedema measures and frequency of CT scans. Hence, a comparison with a protocol-based non-ICP plan is unavoidable. I do agree that the protocol may vary from region to region and the one we have compared with is a fairly standard regime followed in this part of the world.

It has also been stated that the decision for the decompressive craniectomy in the ICP group was taken only when the raised ICP was not amenable to medical management after appropriate time depending on the mode of medical interven- tion. This time period regarding response to medical management is fairly pharmacologically standardized and does not require further elaboration.

In the non-intracranial pressure-monitoring group, mannitol and hypertonic saline where used depending on the admitting consultants' choice. Moreover, whenever mannitol was stopped due to renal impairment, other measures including decompressive craniectomy was resorted to depending on the clinical status and radiological finding of the patient the details of which are beyond the scope or purpose of the article.

ICP re-zeroing generally does not take more than $15 \mathrm{~min}$ and none of the transducer-related problems necessitated patients being off monitoring for more than 10 to $15 \mathrm{~min}$ and the one case of transducer-related haemorrhage was not clinically relevant in terms of haemorrhage volume.

Once again, I wish to thank the author of the appraisal and would hope that better designed studies will be available in future to bridge the gaps.
This article is part of the Topical Collection on Brain trauma

Sudish Karunakaran sudishk@gmail.com

1 Department of Neurosurgery, Medical Trust Hospital, M.G. Road, Kochi, Kerala 682020, India 